\title{
PP009 Improving bacterial cellulose for blood vessel replacement: functionalization with a chimeric protein containing a cellulose-binding module $\&$ an adhesion peptide
}

\author{
F Andrade ${ }^{1}$, R Costa 2 , R Soares², L Dominguesªnd M Gama ${ }^{1}$ \\ ${ }^{1}$ University of Minho, Institute for Biotechnology and Bioengineering - Centre of Biological Engineering, \\ Braga, Portugal \\ ${ }^{2}$ University of Porto, Faculty of Medicine - Department of Biochemistry, Porto, Portugal
}

\section{Background}

Cardiovascular disease is the main cause of mortality in the USA and Western countries, leading to a demand for new materials that can be used as substitutes for blood vessels. Bacterial cellulose (BC) is a material with excellent biocompatibility and mechanical properties, thus holding great potential for arterial replacement. To improve the adhesion of human microvascular endothelial cells (HMECs) to BC, chimeric proteins containing a cellulose-binding module (CBM) and an adhesion peptide (RGD or GRGDY) were produced and used to cover the cellulose fibers. The effects of these proteins were studied.

\section{Materials \& methods}

The attachment/viability and apoptosis were measured by the MTS and TUNEL methods, respectively. Confocal microscopy was used to analyze the morphology of microvascular cells grown on $\mathrm{BC}$ membrane. The visualization of cellulose fibers was also possible once the recombinant protein RGD-CBM was chemically linked to the fluorescein isothiocyanate. The formation of capillary-like structures by the endothelial cells was observed with optical microscopy. To evaluate the RGD effect on the migration of the cells through the $\mathrm{BC}$, a migration chamber and an attractant were used to stimulate the cells to grow into the BC.

\section{Results}

The results of the MTS test demonstrated that the recombinant proteins containing adhesion sequences were able to increase significantly the attachment of HMEC to BC surfaces, and TUNEL assay showed that after $24 \mathrm{~h}$ of adhesion, independently of the treatment, cells remained viable on the BC pellicle, even when less cells adhered to cellulose treated with the CBM or buffer comparing to RGD. The images by confocal microscopy showed that the cells on the BC treated with the RGD presented a more elongated morphology and proliferated on the pellicle, forming a confluent cell layer on the membrane after 7 days. The results showed that the RGD decreased the ingrowth of the HMEC cells through the $\mathrm{BC}$ and also stimulated the early assembly of endothelial cells into capillary-like structures.

\section{Conclusions}

In this work, a new approach was successfully developed to functionalize the $\mathrm{BC}$. The use of recombinant proteins containing a CBM domain, exhibiting high affinity and specificity for cellulose surfaces, allows the control on the interaction of this material with cells. The CBM may virtually be combined to any biologically active protein for the modification of cellulose-based materials, for in vitro or in vivo applications.

\section{PP010} A novel light-emitting photosensitive technique to select \& purify cell populations for use in diagnostics \& therapeutic applications

\section{K Andrews ${ }^{1}$ and J Hunt ${ }^{1}$ \\ ${ }^{1}$ University of Liverpool, UKCTE, UKBioTEC, Clinical Engineering, School of Clinical Sciences, LINSET, Liverpool, UK}

\section{Objectives}

Regenerative medicine increasingly requires accurate diagnostic techniques combined with cell-based therapies. However, cells sought for these applications are often rare in number and difficult to obtain/isolate, resulting in patients undergoing complicated, expensive and invasive procedures. These methods are time consuming with variable clinical success rates, largely due to poor or nonexistent cell-purification techniques.

The aim of this research was to develop a novel light-emitting photosensitive technique, based on patent US 7354733B2, capable of selecting targeted cells for diagnostics and eventual use in specific therapeutic applications. 